\title{
Effect of an Ecological Attentional Bias Modification towards Positive Cues on Positive Affect and Well-Being
}

\author{
Antoine Lecat, Fanny Weytens, Moïra Mikolajczak \\ Research Institute for Psychological Sciences, UCLouvain, Louvain-la-Neuve, Belgium \\ Email: moira.mikolajczak@uclouvain.be
}

How to cite this paper: Lecat, A., Weytens, F., \& Mikolajczak, M. (2019). Effect of an Ecological Attentional Bias Modification towards Positive Cues on Positive Affect and Well-Being. Psychology, 10, 648-666. https://doi.org/10.4236/psych.2019.105041

Received: February 18, 2019

Accepted: April 7, 2019

Published: April 10, 2019

Copyright $\odot 2019$ by author(s) and Scientific Research Publishing Inc. This work is licensed under the Creative Commons Attribution International License (CC BY 4.0).

http://creativecommons.org/licenses/by/4.0/

\section{cc) (i) Open Access}

\begin{abstract}
This article aims to investigate whether a simple attentional training computer task aimed at increasing attention towards positive stimuli can increase positive affects and provide the same benefits on mental health than classic positive psychology exercises. We addressed this issue by experimentally manipulating selective attentional response to positive information and examining its impact on the level of individuals' positive affects and subsequent well-being. Results show that we were not able to induce an attentional bias towards positive cues in all participants, suggesting that some people are more responsive to positive attentional training than others. However, responsive subjects showed an increase in well-being that was proportional to the positive bias induced. Taken together, our results suggest that typical positive psychology exercises currently remain the "gold standard".
\end{abstract}

\section{Keywords}

Attentional Bias Modification, Attentional Training, Well-Being, Positive Affect, Dot Probe Task, Computer-Task Experiment, Positive Psychology, Happiness, Positive and Negative Stimuli, Intervention

\section{Introduction}

It is well-established in literature that vulnerability to negative emotion is linked to attentional bias toward negative and aversive stimuli and information (e.g. MacLeod, Rutherford, Campbell, Ebsworthy, \& Holker, 2002). From this observation, we could assume that inducing an attentional bias toward positive cues can have an impact on emotions by reducing negative affects or increasing positive affects. But can a simple computer game have the same impact on individual 
well-being than typical positive psychology interventions (e.g. practising gratitude or optimism, "the three good things" exercise, the "best possible self" exercise)? This question, which may seem provocative at first sight, is worth to be asked with regard to the therapeutic benefits recently obtained with computer interventions in the treatment of emotional disorders such as social anxiety. Relying on the fact that what individuals attend to clearly shapes their affective experience of an event (Wadlinger \& Isaacowitz, 2011) and that anxious individuals preferentially allocate their attention to threatening stimuli, MacLeod et al. (2002) sought to change their attentional preferences (also called "attentional bias"). By using computerized task to reallocate attention, they made anxious individuals less likely to pay attention to negative stimuli and therefore less likely to suffer from anxiety symptoms.

Based on evidence that attentional processes are at least partially malleable and that changes in attentional focus may change the emotional experience of a situation, we decided to test whether it is possible to bias individual's attention towards positive stimuli. Because paying attention to positive stimuli in the environment favours the emergence of positive emotions (Wadlinger \& Isaacowitz, 2011), and because positive emotions in turn increase attention to positive stimuli (Tamir \& Robinson, 2007), the use of an effective attentional training could be an easy way for people to get into a positive attentional/mood spiral. We were therefore interested in evaluating the impact of the attentional bias modification on individual's emotions and well-being. If this technique proves to be effective, the computerized task could become an alternative or a complementary tool to the current panel of well-being enhancing strategies developed by researchers in positive psychology.

Studies about attentional bias modification have received ever increasing attention over the last decade, but they mainly focused on the reduction of the bias towards negative information. Studies focusing on bias towards positive information are comparatively scarce, which explains the present study.

\section{Attentional Processes and Mood Disorders}

As attentional mechanisms play a crucial role in the emotion generation process, people having a higher (automatic or controlled) propensity to pay attention to negative information are more likely to experience negative affects (Compton, 2000). Because a systematic attentional bias towards negative cues may lead to persistent negative mood states, some authors have suggested that this propensity to attend to negative emotions plays a central role in the aetiology and in the maintenance of mental illnesses such as anxiety and depression (e.g. Beck, 1976).

The use of the visual "dot-probe paradigm" proposed by MacLeod, Mathews and Tata (1986) has enabled the investigation of these potential differences in attentional processes in clinical and non-clinical population. The dot probe paradigm is a computer-based task in which two emotional (or one emotional and one neutral) stimuli (e.g. words or faces) are briefly presented on the screen and 
immediately followed by a probe (e.g. an arrow) to which the participant must respond by pressing, as quickly as possible, on the corresponding key of the keyboard (e.g. "s" or " $m$ " letters). The basic assumption behind this task is that response time will be shorter if the participant's attention is already focused at the location in which the probe appears (MacLeod et al., 1986). As a consequence, an attentional bias toward negative (vs. neutral stimuli) is shown when participants respond to probe that replace negative stimuli faster than those replace the neutral one.

Thanks to this paradigm, research has now consistently confirmed that individuals suffering from mood disorders such as anxiety and depression are indeed subject to specific attentional bias (for review, see Mathews \& MacLeod, 2005; Mogg \& Bradley, 1998; Williams, Watts, MacLeod, \& Mathews, 1997). If the use of the classical dot-probe paradigm has shown links between mood disorders and attentional biases, this technique does not allow to demonstrate a causal link between these processes (i.e. that the attentional bias causes mood disorders). The only way to investigate whether the relationship is causal (or etiologic) is to manipulate attentional biases and to observe the effect of this manipulation on mood. This manipulation can occur towards negative cues or positive cues. First, we will develop attentional bias towards negative cues.

\subsection{Manipulation of Attention towards Negative Cues and Subsequent Changes in Negative Affectivity}

In order to experimentally investigate the hypothesis of a causal link between attentional processes and subsequent negative emotions, MacLeod and colleagues (2002) tried to change individuals' attentional preferences. By adapting the classic dot probe paradigm, they created an attentional bias training towards threatening stimuli. In this task, participants were asked to respond to a visual probe that consistently appeared following threatening-related or neutral words, depending on the participant's condition ("biased towards threat" vs. "control"). In the "biased towards threat" group, the frequent apparition of the probe in the side of threatening words created a contingency leading to the development of an increased (or biased) attentional response to threat: over the training, participants in the "attend to threat" group progressively preferentially allocated their attention towards the side of the threatening words. By using this task, MacLeod and colleagues (2002) successfully induced a bias towards negative stimuli in their experimental group. Through this training, they demonstrated that increased attention towards negative cues is associated to higher levels of negative affects and depression in reaction to a subsequent negative stimulus (here, an anagram stress task).

This seminal study strongly stimulated subsequent research on attentional reallocation trainings. If attentional biases could be increased with a simple computer task, they could possibly be decreased in the same way. The study of the potential benefits of this new treatment quickly became a "hot topic" in the experimental psychopathology literature. Based on the idea that anxiety, social 
phobia and mood disorders are caused and sustained by an attentional bias towards negative stimuli, dozens of papers about attentional reallocation trainings have flourished. Taken together, these studies have shown a medium effect of trainings designed to reduce attentional bias towards negative stimuli (e.g. threatening words, rejecting or disgusted faces) which created a small to medium related decrease in negatives emotions in healthy, anxious and social phobic individuals (for reviews, see Bar-Haim, 2010; Hakamata et al., 2010; Hallion \& Ruscio, 2011). Among these researches, a few studies looked into the attentional bias towards positive stimuli.

\subsection{Manipulation of Attention towards Positives Cues and Subsequent Changes in Negative Affectivity}

Among studies aiming at investigating the impact of attention disengagement from negative stimuli, some research also investigated the impact of a training designed to bias attention towards positive stimuli. Even if not every study found an impact of the attentional training on attentional bias towards positive stimuli (e.g. Boettcher et al., 2013; Kruijt, Putman, \& Van der Does, 2013), some of them did, through dot probe tasks using emotional words (e.g. Taylor, Bomyea, \& Amir, 2011; Wadlinger \& Isaacowitz, 2008) or emotional faces (e.g. Li, Tan, Qian, \& Liu, 2008). In 2007, Dandeneau, Baldwin, Baccus, Sakellaropoulo, \& Pruessner (2007) also successfully modified individuals' attentional bias through another kind of training, based on the same principle of attention redirection. In this paradigm, which is a visual search task, individual were asked to find a smiling face within a $4 \times 4$ matrix of frowning, socially rejecting, faces (Dandeneau \& Baldwin, 2004). After having undergone this training for several trials $(\mathrm{n}=112)$, individuals showed a modified attentional bias (with higher propensity to look at positive cues than before the training) and a decreased level of stress.

To date, most of the studies that investigated the benefits of trainings towards positive stimuli were interested in its impact on negative affectivity markers such as anxiety and negative emotions. Li, Tan, Qian and Liu (2008) showed for example that a 7-day attentional dot-probe training towards positive faces led to decreased attention towards negative faces and decreased anxiety in social interactions in social phobic individuals. In a 2009 study, Johnson demonstrated that people trained to attend to positive information, using a task similar to Macleod et al. (2002) with positive and negative stimuli, experienced lower levels of frustration than a control group after being exposed to a stressor (Johnson, 2009). In the same line, Dandeneau and his colleagues showed that a multi-session (4 or 5 days) attentional training towards positive faces led to lower perceived and objective (through cortisol measurement) stress among students and telemarketers. Although these authors have also shown a positive impact of their training on students' self-perceived academic competencies and self-esteem and on telemarketers' self-confidence and sales performance, they have not extended their investigation to variables such as positive affect or the level of satisfaction with life of their participants (Dandeneau et al., 2007, study 3b). Furthermore, even if 
this research demonstrated that training attention to positive stimuli helped individuals to reduce their negative emotions, unfortunately, the authors did not investigate whether these improvements were mediated by a generalized preference towards positive information after the training (Wadlinger \& Isaacowitz, 2008).

A recent study, using the same positive attentional training (with happy and angry faces) with anxious children, showed a decrease in clinician evaluation of children' anxious symptoms, but was unable to demonstrate that these improvements were mediated by changes in attentional bias in their small sample ( $\mathrm{n}=37$; Waters, Pittaway, Mogg, Bradley, \& Pine, 2013). However, these authors interestingly showed that the bias learned on specific material (a particular set of pictures) was transposable to different material of the same valence (i.e. other set pictures). This idea is congruent with Wadlinger and Isaacowitz (2008)'s finding that attentional preferences toward positive information can be successfully trained and that these preferences can be effectively generalized to other visual information. Taken together, these studies, investigating the impact of attentional trainings towards positive or negative cues, confirmed the causal role of attention on subsequent negative affects.

\subsection{Manipulation of Attention towards Positive Cues and Subsequent Changes in Positive Affectivity}

Although many studies have investigated the links between attentional bias and negative affectivity during the last fifteen years, interest for attentional biases and their impact on positive affectivity and well-being have remained surprisingly low in the literature (Grafton, Ang, \& MacLeod, 2012). However, if an increased attentional bias towards cues from a specific emotional valence causes a higher disposition to experience the corresponding emotional state, as proposed by Wadlinger and Isaacowtiz (2011), attentional training towards positive information may represent a mean to increase individuals' positive affectivity.

In order to investigate this possibility, Grafton, Ang and MacLeod (2012) ran the first experimental study that assessed the impact of an attentional training towards positive stimuli on positive affectivity. Using a design paralleling MacLeod and colleagues' seminal study (2002), these authors assigned their participants to a single-session dot probe attentional training task. While participants of the experimental group were biased towards positive words (vs. neutral words), those from control group were exposed to a no contingency condition (equal probability of apparition of the probe next to positive and neutral cues). Response times' evolution suggest that participants in the experimental group became more attentive to positive emotional cues than participants in the control group after the training, but this difference was not statistically significant. Interestingly, state positive affectivity (assessed with the PA scale of the PANAS, Watson, Clark, \& Tellegen, 1988) measured right after the training showed no differences between the groups. However, even if the state positive affectivity was not influenced by the experimental manipulation, the subsequent reaction of 
participants to a positive event was. The level of positive affectivity of biased-towards-positive participants was significantly higher than their control counterparts after a positive feedback on their performance to an anagram task. Even if these results suggest that individual differences in attentional bias towards positive cues can causally contribute to positive affectivity, this first study also reported difficulties to induce a bias towards positives cues, despite the use of a methodology that was proven efficient to reduce an attentional bias toward the negative ones.

Based on the foregoing results, Grafton et al. (2012) suggested several avenues for future research. Because they tested the impact of an enhanced attentional bias towards positive information on reactivity to a laboratory situation (i.e. the anagram task), they invited researchers to assess the potential of such attentional training on individuals' emotional response to naturalistic situations. They therefore suggested to induce a more durable attentional bias through multiple attentional training sessions and to investigate the effects of such training on more distal variables of positive affectivity, representative of participants' "real life" affective experience and well-being. As they pointed, there are good reasons to hope that biased attention may remain effective after initial induction as studies aiming at shifting individuals' attention away from negative cues led to significant changes in attentional processes and real-world negatives affects up to three months after the end of the training (e.g. Amir, Beard, Burns, \& Bomyea, 2009a; Schmidt, Richey, Buckner, \& Timpano, 2009).

\section{The Present Study}

In line with Grafton et al.' (2012) suggestion, we created an experimental design aiming at investigating the effects of a longer (5 sessions on 7 days rather than one single session) attentional training towards positive cues on subsequent individual's positive affectivity and well-being (assessed 1 day and 2 weeks after training rather than just after), compared to a non-preferentially valenced condition (50\% towards positive cues and 50\% towards negative cues). Because our aim was to induce a bias towards positive cues that would be easily transposable to individuals' real-life attentional processes, we adapted the training used by Grafton et al. (2012) into a more ecological version. Rather than using positive and neutral words as cues, we preferred to use pictures of everyday life people and objects (e.g. gift, smiling baby or rainbow for positive cues; and rat, garbage or stains for negative cues). In order to train participants to catch attractive stimuli in real-life situations, four stimuli (two positive and two negative, each of them in a specific quarter of the image) were simultaneously placed in real-life backgrounds (e.g. in the garden, in the kitchen or inside of a car). The detailed method is presented hereafter.

\subsection{Method}

\subsubsection{Participants}

The study, reviewed and approved by the Research Ethics Board of the Psychol- 
ogy Department of the University of Louvain, was presented to the participants as a "Catch Me Game". Opportunity sampling was used to find participants by publishing public notice in the university hall. All students that have shown interest during the recruitment week were accepted in the sample. Participants were a total of sixty-four undergraduate students who participated for extra course credit and who were randomly assigned in experimental or control condition. 5 participants from the experimental group and 6 from the control failed to complete all the training sessions and their results where therefore excluded from the analyses. So, analyses concerning the first post-test (the day after the last training) include data of a final sample $(n=53)$ consisting of twenty-seven students in the experimental condition ( 23 women, $M=20.48$ years, $S D=1.72$ ) and twenty-six in the control ( 18 women, $\mathrm{M}=20.15$ years, $\mathrm{SD}=1.16$ ). At the two weeks post-test, only 24 participants in the experimental (21 women, $\mathrm{M}=$ 20.29 years, $S D=1.73$ ) and 20 in the control condition ( 13 women, $M=20.20$ years, $\mathrm{SD}=1.15)$ completed the questionnaires.

\subsubsection{Procedure}

In order to complete the pre-test, the participants came the first day (on Tuesday) in a computer room for a group session. We explained them the study procedure and they signed an informed consent. Then, they were asked to complete the computerized attentional bias measure (see Materials below), to fill questionnaires about personality, mood and happiness (see Measures below) and to start the first session of the Catch Me Game (i.e. the attentional bias training; see Materials) by logging in a personal session of the software, matched with their condition. At the end of this first session, participants were asked to come once a day to the computer room, whenever they wanted, during the four stipulated days (Wednesday, Thursday, Friday of the first week and Monday of the second week), in order to play to the game. Each gaming (i.e. training) session lasted for approximately 15 minutes. On the day after the last training (Tuesday, week 2, see Figure 1 for a representation of the chronology of the study), participants came back to the computer room for the second group session. They completed questionnaires and the attentional bias measure again. Finally, two weeks after the last training session, they filled a last questionnaire at home (sent by email).

\subsubsection{Materials}

1) Experimental materials

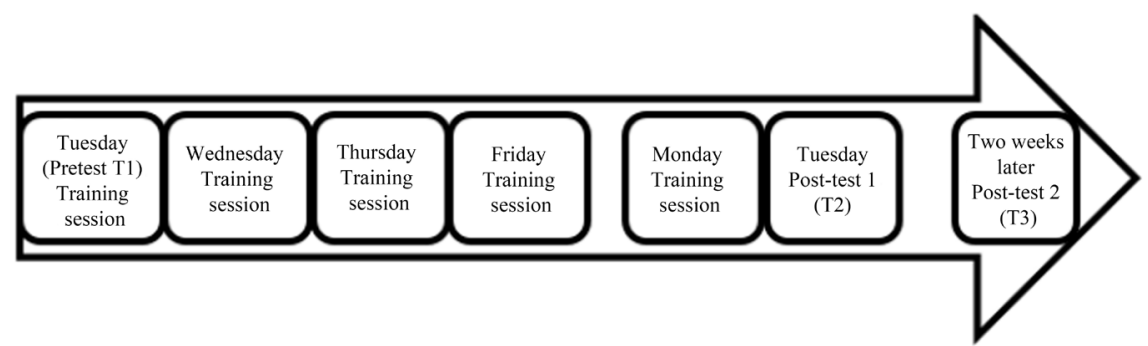

Figure 1. Chronology of the study. 
For the attentional bias measure and the attentional bias training, computer-tasks were used. For each of these tasks, images, presented on a computer screen, consisted in a neutral "daily life" background with realistically embedded emotionally positive and negative stimuli (see Figure 2). All the attentional bias measures and attentional bias trainings were run on personal computers using Java $^{\text {TM }}$ Platform SE binary on a 17 -inch $(432 \mathrm{~mm})$ Dell M782p monitor to display stimuli and record response times. Each background was adjusted in $960 \times$ 720 pixels $(22.9 \times 17.6 \mathrm{~cm})$. Participants sat about $70 \mathrm{~cm}$ away from the computer screen. Stimuli were about $2-4 \mathrm{~cm}$ in height and $3-5 \mathrm{~cm}$ in width, depending on the realistic location they could have in the background. More details are provided in the "Attentional bias measure" and "Attentional training" sections below.

\section{2) Self-reported measures}

Personality factors were measured via the International Personality Item Pool (IPIP; Goldberg, 1992). We used the 50-item version of the IPIP which comprises 10 items for each of the Big-Five personality factors: Extraversion (tendency to be outgoing and sociable vs. reserved and solitary), Agreeableness (tendency to be cooperative and friendly vs. analytical and suspicious), Conscientiousness (tendency to be organized and show self-discipline vs. easy-going), Emotional Stability (tendency to be confident and secure vs. to experience unpleasant emotions easily) and Intellect (tendency to be curious, inventive and to appreciate art vs. cautious and consistent). Each item was rated on a 5-point Likert scale from 1 "very inaccurate" to 5 "very accurate". The internal consistency $(\alpha)$ at T1 is 0.84 for extraversion, 0.70 for agreeableness, 0.74 for conscientiousness, 0.69 for emotional stability and 0.71 for intellect.

Positive and negative affects were assessed by the Scale of Positive and Negative Experience (SPANE; Diener, Wirtz, Biswas-Diener, Tov, Kim-Prieto, Choi \& Oishi, 2009) which is a 12-item self-reported questionnaire assessing positive

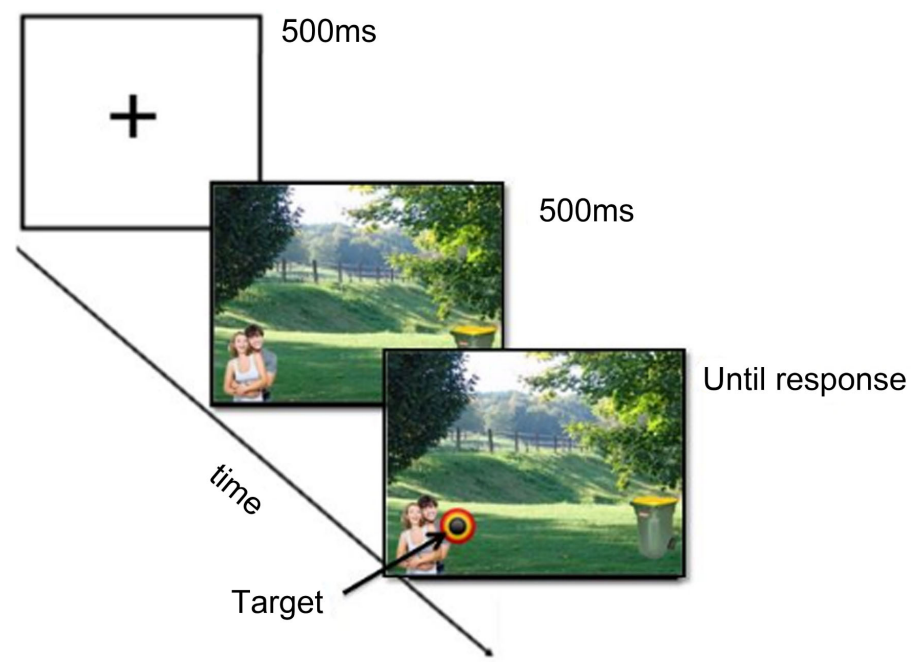

Figure 2. An illustration of a positive trial in the attentional bias measure (smiling couple on the left vs. trash bin on the right). 
and negative feelings and emotions over the past week. Three of the six items of each valence assess general feelings (e.g. "positive", "negative") and the three others identify specific emotions (e.g. "joy", "sadness"). The SPANE was preferred to the PANAS (Watson, Clark, \& Tellegen, 1988) because it assesses a more general conception of emotional well-being and ill-being than the PANAS (Diener et al., 2010). The internal consistency $(\alpha)$ for positive items was 0.92 at $\mathrm{T} 1,0.90$ at $\mathrm{T} 2$ and 0.92 at $\mathrm{T} 3$ and the internal consistency $(\alpha)$ for negative items was 0.78 at $\mathrm{T} 1,0.84$ at $\mathrm{T} 2$ and 0.81 at $\mathrm{T} 3$.

Subjective Happiness was measured using the well-validated Subjective Happiness Scale (SHS; Lyubomirsky \& Lepper, 1999). This questionnaire provides a global, subjective assessment of whether the respondent considers himself/herself as a happy or an unhappy person, via four items rated on a 7-point Likert scale (e.g. "Compared with most of my peers, I consider myself" from 1 = not a very happy person to $7=$ a very happy person). The internal consistency $(\alpha)$ was 0.78 at $\mathrm{T} 1$, 0.77 at $\mathrm{T} 2$ and 0.87 at $\mathrm{T} 3$.

Life Satisfaction was assessed with the Satisfaction with Life Scale (SWLS; Diener, Emmons, Larsen, \& Griffin, 1985). This scale comprises five items (e.g. "So far I have gotten the important things I want in life") rated on a 7-point scale (from $1=$ strongly disagree to $7=$ strongly agree). The internal consistency $(\alpha)$ was 0.80 at $\mathrm{T} 1,0.80$ at $\mathrm{T} 2$ and 0.82 at $\mathrm{T} 3$ in our sample.

Flourishing was assessed through the Flourishing Scale (FS; Diener, Wirtz, Biswas-Diener, Tov, Kim-Prieto, Choi, \& Oishi, 2010), which is an 8-item scale (e.g. "I am engaged and interested in my daily activities") evaluating self-perceived flourishing in important areas such as relationships, self-esteem and purpose. Participants had to rate on a 7-point scale the degree of agreement (from $1=$ strongly disagree to $7=$ strongly agree). The internal consistency $(\alpha)$ was 0.72 at $\mathrm{T} 1,0.77$ at $\mathrm{T} 2$ and 0.77 at $\mathrm{T} 3$.

Somatic complaints were assessed through a short version of the Pennebaker Inventory of Limbic Languidness (PILL; Pennebaker, 1982; Nelis et al., 2011, short version). The abbreviated scale consists of a list of the 29 most common physical symptoms (e.g., headache, stomach-ache, sleep problems, cramps). Participants had to rate on a 5-point scale the frequency with which they experienced each symptoms/sensation during the past week $(1=$ never or nearly never, 2 = sometimes, 3 = frequently, $4=$ very frequently, $5=$ almost always). The internal consistency $(\alpha)$ was 0.82 at T1, 0.85 at T2 and 0.88 at T3 in our sample.

Optimism was measured by the Life Orientation Test - Revised (LOT-R; Scheier, Carver, \& Bridge, 1994), which is a self-reported questionnaire assessing individual differences in generalized optimism versus pessimism. Participants had to rate 10 items (e.g. "In uncertain times, I usually expect the best") on a 5 -point scale (from 1 = "I agree a lot" to 5 = "I disagree a lot"). The internal consistency $(\alpha)$ was 0.76 at $\mathrm{T} 1,0.77$ at $\mathrm{T} 2$ and 0.82 at $\mathrm{T} 3$.

Positive Thinking was assessed through the Positive Thinking Scale (PTS; Diener, Wirtz, Biswas-Diener, Tov, Kim-Prieto, Choi, \& Oishi, 2010). The PTS is a 
22-item self-reported questionnaire measuring positive and negative thinking over the past week, with 11 items representing positive thinking (e.g. "I see the good in most people") and 11 items representing negative thinking (e.g. "When I think of myself, I think of many shortcomings"). In the original version, all the items were dichotomous and were responded on a yes-no format. In this study, we created a 7-item Likert scale for each item in order to increase the scale sensitivity (from $1=$ "I totally disagree with this sentence" to " 7 = "I totally agree with this sentence"). The internal consistency $(\alpha)$ was 0.65 at T1, 0.58 at T2 and 0.86 at $\mathrm{T} 3$.

Depression was measured via the short version of the Centre for Epidemiologic Studies Depression Scale (CES-D 10; Andresen, 1994). The CES-D 10 is a 10-item self-reported scale assessing depressive symptoms on a 5-point Likert scale (from 1 = "Never or nearly never" to $5=$ "All the time, or almost all the time"). The internal consistency ( $\alpha$ ) was 0.78 at T1, 0.75 at T2 and 0.75 at T3.

\section{3) Attentional bias measure}

In this computerized-task, a fixation cross was presented at the centre of a computer screen for 500 milliseconds (ms). Next, a daily life background appeared with two embedded (and realistically placed) stimuli. One was emotionally positive (e.g. a smiling couple, see in Figure 2, left) and the other one was negative (e.g. a trash bin, see in Figure 2, right). Selected stimuli were daily-life objects, animals and persons. The valence of the pictures was pre-tested by 6 students on a seven-point Likert ( $1=$ negative and $7=$ positive $)$ scale. Pictures that received a mean score of 6 or more, and equal or less than 2, were kept. In the attentional bias measure, stimuli of each valence were placed on opposite sides (left/right) of the background and their location was counterbalanced during the entire task. After $500 \mathrm{~ms}$, a target appeared near one of the stimuli. Participants were asked to click on the target as quickly as possible. The next trial began immediately after participant's click, with the fixation cross.

This task comprised a total of 48 trials among which the first 3 were designated example trials and the following 45 were real trials for bias measurement. For participants of both groups, targets appeared with equal frequency near emotionally positive or negative stimuli. Cumulative response times for "near positive stimulus" and "near negative stimulus" were separately calculated ("response time for positive cues", RT pos; and "response time for negative cues", RT neg). An attentional bias score was then computed (Attention Bias = RT for "near negative" - RT for "near positive"), in order to evaluate the preferential allocation of attention of participants toward stimuli of one the valences.

4) Attentional training

After the attentional bias measure, in a second task, a fixation cross was also presented at the centre of the computer screen for $500 \mathrm{~ms}$. Right after, a daily life background appeared with four embedded (and realistically placed) stimuli (two emotionally positive and two emotionally negative, see Figure 3 for an example). Positively and negatively valenced stimuli were placed left/right, bottom/up or 
diagonally (see Figure 3) and their locations were counterbalanced during the entire task (see Figure 4 for an example of each possible configuration). A target (see Figure 3) appeared simultaneously on the background. The image remained on the screen until the participant made a click on the right place. The next trial began immediately with the fixation cross after the participant's click. This task comprised a total of 335 trials/session. Thus the total number of trials completed by the participants is 1675 .

\subsubsection{Conditions}

During the attentional training, for participants in the experimental group, ninety percent of the targets appeared near a positive stimuli (that is, in a positive area, identified by a " + " in Figure 4). By doing so, we expected to induce an unconscious attentional bias towards positive stimuli. For participants in the no-contingency control group, targets appeared near emotionally positive or negative stimuli with equal frequency $(50 \%-50 \%)$.

\subsection{Results}

\subsubsection{Pre-Test Comparisons}

Firstly, we examined potential differences between the groups on gender, age

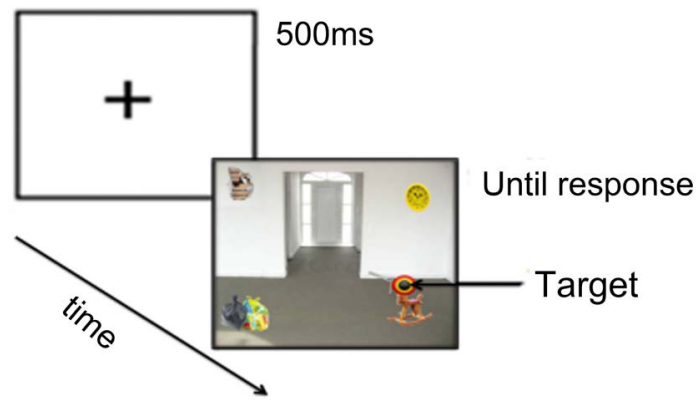

Figure 3. An illustration of a positive trial in the "Catch me Game" (i.e. the attentional bias training).
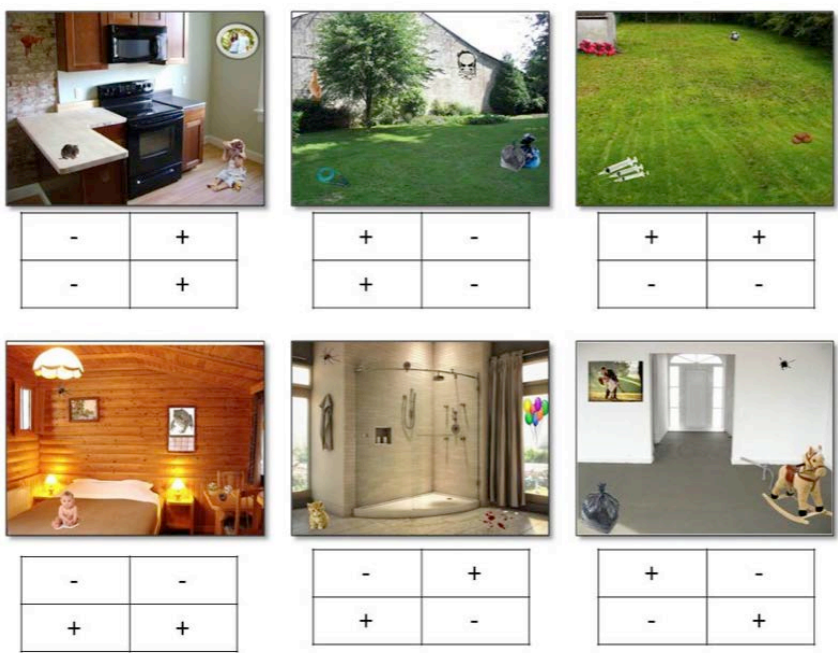

Figure 4. Examples of stimuli combinations in every-day life backgrounds. 
and personality facets in order to verify that no baseline difference existed between the groups on these characteristics. Neither the chi-square test performed on gender, $X^{2}(1,53)=1.925, p=0.17$, nor the independent $T$-tests performed on mean ages of the groups, $t(51)=0.811, p=0.42$ was significant. Comparison on personality facets revealed no difference between groups ( $p$ 's ranging from 0.171 to 0.994 ), except for conscientiousness $t(51)=2.596, p=0.012$. Despite random allocation of participants in the groups, participants of the experimental group appeared to be more conscientious $(\mathrm{M}=37,11, \mathrm{SD}=5.96)$ than those in the control group $(M=33,27, S D=5.41)$. However, this difference had no effect on the training rate and compliance.

Secondly, we compared groups response times for positive and negative trials in the attention bias measure at pre-test to ensure that there were no between-group differences before the training. Results of independent $T$-tests between the groups were neither significant for the positive stimuli response time nor for the negative stimuli response time (see Table 1), indicating that there were no significant differences in attentional preferences between the two groups at pre-test.

Thirdly, as already explained, we computed a bias score on the basis of these indicators, corresponding to the difference between response time for positive cues and negative cues (Attentional bias $=\mathrm{RT}$ negative $-\mathrm{RT}$ positive). Given the

Table 1. Means (and standard deviations) at each time for each group, pre-test comparisons and analysis of variance.

\begin{tabular}{|c|c|c|c|c|c|c|c|c|c|c|c|c|}
\hline & \multicolumn{2}{|c|}{ Experimental group } & \multicolumn{3}{|c|}{ Control group } & \multicolumn{3}{|c|}{ Pre-test comparisons } & \multicolumn{4}{|c|}{ ANOVA's } \\
\hline & $\begin{array}{c}\mathrm{T} 1 \\
\mathrm{M}(\mathrm{SD}) \\
\mathrm{N}=27\end{array}$ & $\begin{array}{c}\mathrm{T} 2 \\
\mathrm{M}(\mathrm{SD}) \\
\mathrm{N}=27\end{array}$ & $\begin{array}{c}\text { T3 } \\
\mathrm{M}(\mathrm{SD}) \\
\mathrm{N}=24\end{array}$ & $\begin{array}{c}\mathrm{T} 1 \\
\mathrm{M}(\mathrm{SD}) \\
\mathrm{N}=26\end{array}$ & $\begin{array}{c}\mathrm{T} 2 \\
\mathrm{M}(\mathrm{SD}) \\
\mathrm{N}=26\end{array}$ & $\begin{array}{c}\mathrm{T} 3 \\
\mathrm{M}(\mathrm{SD}) \\
\mathrm{N}=20\end{array}$ & $t(51)$ & $p$-value & $\begin{array}{l}\text { T1 vs. T2 } \\
F(1,51)\end{array}$ & $p$-value & $\begin{array}{l}\text { T1 vs. T3 } \\
\mathrm{F}(1,42)\end{array}$ & $p$-value \\
\hline $\mathrm{RT}$ (pos) & $1131(181)$ & $941(125)$ & I & $1125(144)$ & $976(155)$ & l & 0.132 & 0.896 & 0.622 & 0.434 & / & / \\
\hline RT (neg) & $1138(201)$ & 949 (105) & I & 1128 (159) & $971(133)$ & I & 0.203 & 0.840 & 0.555 & 0.460 & I & I \\
\hline $\begin{array}{l}\text { Attentional } \\
\text { bias }\end{array}$ & $7.08(137)$ & $7.84(86)$ & I & $2.86(114)$ & $-4.28(80)$ & I & 0.121 & 0.904 & -0.022 & 0.884 & I & l \\
\hline $\begin{array}{l}\text { Positive } \\
\text { affects }\end{array}$ & $4.06(0.71)$ & $4.05(0.60)$ & $4.20(0.71)$ & $4.30(0.86)$ & $25.77(4.30)$ & $4.12(0.86)$ & -1.081 & 0.285 & 0.017 & 0.897 & 1.847 & 0.181 \\
\hline $\begin{array}{l}\text { Negative } \\
\text { affects }\end{array}$ & $2.70(0.66)$ & $2.45(0.67)$ & $2.70(0.70)$ & $2.39(0.66)$ & $2.35(0.75)$ & $2.34(0.75)$ & 1.730 & 0.090 & 1.028 & 0.315 & 0.006 & 0.938 \\
\hline $\begin{array}{l}\text { Subjective } \\
\text { happiness }\end{array}$ & $4.66(0.99)$ & $4.81(0.75)$ & $4.93(0.91)$ & $4.88(1.20)$ & $4.97(1.18)$ & $5.03(1.29)$ & -0.755 & 0.454 & 0.131 & 0.719 & 0.057 & 0.813 \\
\hline $\begin{array}{l}\text { Satisfaction } \\
\text { with life }\end{array}$ & $5.05(1.10)$ & $5.28(0.94)$ & $5.33(0.98)$ & $4.93(0.99)$ & $5.14(0.83)$ & $4.59(0.88)$ & 0.207 & 0.837 & 0.233 & 0.631 & 0.002 & 0.966 \\
\hline Flourishing & $5.61(0.62)$ & $5.55(0.63)$ & $5.49(0.62)$ & $5.61(0.47)$ & $5.56(0.54)$ & $5.53(0.88)$ & 0.004 & 0.997 & 0.010 & 0.922 & 0.319 & 0.575 \\
\hline $\begin{array}{l}\text { Somatic } \\
\text { complaints }\end{array}$ & $1.71(0.34)$ & $1.52(0.32)$ & $1.61(0.40)$ & $1.86(0.39)$ & $1.69(0.36)$ & $1.72(0.36)$ & -1.543 & 0.129 & 0.025 & 0.874 & 0.327 & 0.571 \\
\hline Optimism & $2.06(0.46)$ & $2.16(0.40)$ & $2.18(0.48)$ & $2.08(0.45)$ & $2.12(0.48)$ & $2.14(0.52)$ & -0.172 & 0.864 & 0.557 & 0.459 & 1.653 & 0.206 \\
\hline $\begin{array}{l}\text { Positive } \\
\text { thinking }\end{array}$ & $4.56(0.65)$ & $4.79(0.46)$ & $4.82(0.86)$ & $4.53(0.47)$ & $4.80(0.48)$ & $4.82(0.70)$ & 0.197 & 0.845 & 0.059 & 0.809 & 0.016 & 0.900 \\
\hline Depression & $2.06(0.48)$ & $1.89(0.34)$ & $1.99(0.43)$ & $2.20(0.50)$ & $1.86(0.43)$ & $1.93(0.54)$ & -0.984 & 0.330 & 1.424 & 0.238 & 1.612 & 0.211 \\
\hline
\end{tabular}

$\mathrm{RT}$ is response time between target apparition and click. 
arithmetic manipulation, a positive score indicates that the individual takes shorter to respond to positive cues than to negatives, indicating a bias towards positive cues. On the contrary, a negative score indicates a bias towards negative cues. The comparison of the bias values between the groups at pre-test indicates that there was no significant difference in attentional preferences between the two groups at pretest (see Table 1). A $t$ test performed on mean attentional bias of each group revealed that none of the groups significantly differ from zero at pretest $(t(26)=0.267, p=0.791$ for the experimental group and $t(25)=0.127, p$ $=0.900$ for control group), indicating that individuals in both groups may be considered as "non-biased" before the trainings.

Finally, we compared the groups on each self-reported dependant variable at baseline in order to check for potential between-group differences before the training. As shown in Table 1 , none of the independent $T$-tests comparing the means between the groups was significant. We can therefore conclude that the two groups are statistically equivalent at pre-test on these variables.

\subsubsection{Impact of the Training on Attentional Biases}

Trials with inter-individual outlier response time (more than 3 SDs) were excluded from analyses. Intra-individual outliers (more than 3 SDs above each participant's mean) were also excluded because these trials were considered as non-representative of the individual response tendency (e.g. nose-scratching, sneezing).

The mean response time before and after the training within both groups was calculated, for positive and negative trials, as shown in Table 1 . We then performed a 2 (pre-test and post-test) $\times 2$ (groups: experimental and control) ANOVA for repeated measures in order to test for potential differences in the evolution of response time to positive cues between the groups. Results indicated a significant main effect of time, $\mathrm{F}(1,51)=50.80, p=0.00$, indicating that reaction times decreased between Time 1 and Time 2 in both groups, but no two-factor interaction was found significant (see Table 1), indicating that groups do not show a significantly different evolution between pre- and post-test (in the experimental group, RT Time 2 - RT Time 1 for positive cues: $M=-190 \mathrm{~ms}$, SD = 225; in control group, RT Time 2 - RT Time 1 for positive cues: $\mathrm{M}=-147 \mathrm{~ms}, \mathrm{SD}=$ $156)$ in their reaction time for positive cues. Note that, as the groups differed in conscientiousness at Time 1, we included this variable as a covariate in another run of analyses. This inclusion did not change the conclusion on the time $\mathrm{x}$ group interaction, $\mathrm{F}(1,51)=0.787, p=0.379$.

Another 2 (pre-test and post-test) $\times 2$ (experimental groups) ANOVA for repeated measure was performed on response time for negative cues. A significant main effect of time, $\mathrm{F}(1,51)=50.80, p=0.00$ was found, as for positive cues, probably indicating a practice effect. However, the two-factor interaction was not significant, indicating that groups do not show a significantly different evolution between pre- and post-tests (in the experimental group, RT Time 2 - RT Time 1 for positive cues: $\mathrm{M}=-189 \mathrm{~ms}, \mathrm{SD}=203$; in control group, RT Time 2 - 
RT Time 1 for negative cues: $\mathrm{M}=-153 \mathrm{~ms}, \mathrm{SD}=133$, see Table 1 ). Note that the inclusion of conscientiousness as a covariate did not change the conclusion on the time $\mathrm{x}$ group interaction, $\mathrm{F}(1,51)=1,225, p=0.274$.

\subsubsection{Impact of the Training on the Self-Reported Dependent Variables}

In order to investigate if the training influenced positive affects and the other well-being variables, we conducted separate 2 (pre-test and post-test) $\times 2$ (experimental groups) ANOVAs for each dependent variable (i.e. positive affects, negative affects, subjective happiness, satisfaction with life, self-perceived flourishing, physical symptoms, optimism, positive thinking and depression). As expected given the lack of effect of the training on attentional bias induction, none of these two-factor interactions turned out to be significant (see Table 1), indicating that groups do not statistically show different patterns of evolution on these variables between pre- and post-tests. As the groups were different in conscientiousness at Time 1, we included this variable as a covariate in another run of analyses. This inclusion did not change the conclusion for the time $\mathrm{x}$ group interactions ( $p$ 's ranging from 0.234 to 0.884 ).

Finally, data from the two weeks post-test were also analysed by separate 2 (pre-test and last questionnaire) $\times 2$ (experimental groups) ANOVAs. These analyses reported that there was no significant time $x$ group interaction. The inclusion of Time 1 conscientiousness as a covariate did not change the results (p's ranging from 0.142 to 0.959 ).

\subsubsection{Changes in Attention and Related Changes in Well-Being}

The difficulty to induce a statistically significant bias towards positive cues at the group level does not exclude the possibility that the reallocation of attention training worked better for some individuals and that effects on well-being appear in these people only. In order to test whether changes in attention may have been associated with changes in well-being, we examined the associations between RT differences (RT Time 2- RT Time 1; where a negative result indicates an increased attentional bias) for both positive and negative cues and the evolutions in the dependent variables in the experimental group. Pearson correlations showed associations between RT differences for positive cues and difference in satisfaction with life $(r(27)=-0.483, p=0.011)$, difference in optimism $(r(27)=$ $-0.639, p=0.000)$, and difference in depression $(r(27)=0.535, p=0.004)$, respectively. Thus, the greater the increase in attentional bias towards positive cues (i.e. the more negative the RT difference) the greater the improvement in satisfaction with life, optimism and the reduction in depressive symptoms.

Pearson correlations have also shown associations between RT difference for negative cues and satisfaction with life $(r(27)=-0.545, p=0.003)$, optimism $(r(27)=-0.584, p=0.001)$ and difference in depression $(r(27)=0.568, p=$ 0.002). These correlations must be interpreted in the same direction as RT for positive cues: those with the largest acquired attentional bias towards negative cues (i.e. the more negative the RT difference) are those who experience the largest benefits. 


\subsection{Discussion}

Based on the assumption that increased attention towards specific cues leads to an increase in the disposition to experience the corresponding emotional state (Wadlinger \& Isaacowitz, 2011), this study sought to determine whether an ecological computer-based attentional training towards positive information could lead to further benefits for individuals' well-being. Contrary to our expectations, our results indicated that a five-day attentional training towards positive cues did not lead to a significantly greater decrease in response time for positive cues compared to a no contingency control group. Similarly, our results showed no significant difference between the groups on the evolution of the dependent variables under consideration (positive affects, negative affects, subjective happiness, satisfaction with life, self-perceived flourishing, physical symptoms, optimism, positive thinking and depression). Albeit surprising, these results are consistent with those reported by Grafton et al. (2012). These authors attempted to induce a bias toward positive stimuli using an adapted version of the dot probe task and tested its impact on short-term positive affect. While the reaction times for positive stimuli evolved in the expected direction following the attentional training in their experimental group, Grafton et al. (2012) were not able to show that the reaction times in this group evolved statistically differently from the control group.

How could we explain that both Grafton's team and ours were unable to induce an attentional bias toward positive stimuli while numerous studies report effectiveness of such trainings to reduce or induce bias towards negative stimuli? Firstly, it seems important to point out here the heterogeneity of the results obtained by training programs designed to reduce attentional bias towards negative stimuli and the corresponding negative affects, and in particular, anxiety and depressive symptoms. If very encouraging results have been reported during the 2000s (e.g. Amir et al., 2009b; Amir, Weber, Beard, Bomyea, \& Taylor, 2008; Hazen, Vasey, \& Schmidt, 2009), several replication studies have recently failed to reproduce the results previously obtained (e.g. Boettcher et al., 2013; Bunnell, Beidel, \& Mesa, 2013; Carlbring et al., 2012; Heeren, Reese, McNally, \& Philippot, 2012; Neubauer et al., 2013). A recent meta-analysis highlighted the general inconsistency of the results and concluded to a much smaller effect than previously thought (Hallion \& Ruscio, 2011). Depending on the meta-analysis, the effect of sizes reported on bias change varies, and can range from a medium (Hakamata et al., 2010; 10 articles, 457 participants) to small effect size (Hallion \& Ruscio, 2011; 39 articles, 2591 participants).

The size of the subsequent changes in negative affect also differ: While Hakamata et al. (2010) report an middle effect size of attentional trainings programs on anxiety symptoms, Hallion and Ruscio (2011) report a small effect that become non-existent when correcting for publication bias. In conclusion, literature is not unequivocal on the effectiveness of the attention training programs intended to reduce negative affects. It is also plausible that these kinds of programs 
are less promising than what was originally thought, an opinion that is spreading (Cristea, Kok, \& Cuijpers, 2015; Heeren, Mogoase, Philippot, \& McNally, 2015) and which led scholars such as Emmelkamp (2012) to recommend not to further pursue the investigation of attentional trainings techniques. If Hallion and Ruscio (2011) and Cristea, Kok, \& Cuijpers, 2015 are right that the effect of attentional programs is null when correcting for publication bias, it may explain why we were unable to find results.

\section{Conclusion}

The study presented above is, to our knowledge, the first evaluation of the impact of an ecological bias-toward-positive induction on all comers well-being. If our results suggest that increases in well-being appeared in people of the experimental group in which the decrease of response times for positive stimuli was the greatest following the training, they also showed that our attentional training is not effective for all individuals. Given our inability to induce a significant bias toward positive stimuli in our experimental group, attentional training to positive stimuli does not represent a valuable alternative to the "traditional" techniques proposed in positive psychology literature (for reviews, see Bolier, Haverman, Westerhof, Riper, Smit, \& Bohlmeijer, 2013; Sin \& Lyubomirsky, 2009) in order to increase individual well-being. However, we did not measure the potential of attentional bias modification in addition to more traditional techniques. Practical implications are firstly that, in the current state of knowledge, well-being still cannot be increased durably using automatic processes alone. The actual fast track to well-being improvement remains positive activities and interventions (e.g. Quoidbach, Mikolajczak, \& Gross, 2015; Sin \& Lyubomirsky, 2009). Future studies may show effects of a combination of techniques including attentional bias modification.

\section{Acknowledgements}

We thank Pierre de Neuville and Stéphanie Moreau for their help in images creation and data collection.

\section{Conflicts of Interest}

The authors declare no conflicts of interest regarding the publication of this paper.

\section{References}

Amir, N., Beard, C., Burns, M., \& Bomyea, J. (2009a). Attention Modification Program in Individuals with Generalized Anxiety Disorder. Journal of Abnormal Psychology, 118, 28-33.

Amir, N., Beard, C., Taylor, C. T., Klumpp, H., Elias, J., Burns, M., \& Chen, X. (2009b). Attention Training in Individuals with Generalized Social Phobia: A Randomized Controlled Trial. Journal of Consulting and Clinical Psychology, 77, 961-973.

Amir, N., Weber, G., Beard, C., Bomyea, J., \& Taylor, C. T. (2008). The Effect of a Sin- 
gle-Session Attention Modification Program on Response to a Public-Speaking Challenge in Socially Anxious Individuals. Journal of Abnormal Psychology, 117, 860-868. https://doi.org/10.1037/a0013445

Bar-Haim, Y. (2010). Research Review: Attention Bias Modification (ABM): A Novel Treatment for Anxiety Disorders. Journal of Child Psychology and Psychiatry, 51, 859-870. https://doi.org/10.1111/j.1469-7610.2010.02251.x

Beck, A. T. (1976). Cognitive Therapy and the Emotional Disorders. Penguin.

Boettcher, J., Leek, L., Matson, L., Holmes, E. A., Browning, M., MacLeod, C., Andersen, G., \& Carlbring, P. (2013). Internet-Based Attention Bias Modification for Social Anxiety: A Randomised Controlled Comparison of Training towards Negative and Training towards Positive Cues. PLoS ONE, 8, e71760. https://doi.org/10.1371/journal.pone.0071760

Bolier, L., Haverman, M., Westerhof, G. J., Riper, H., Smit, F., \& Bohlmeijer, E. (2013). Positive Psychology Interventions: A Meta-Analysis of Randomized Controlled Studies. BMC Public Health, 13, 119-139. https://doi.org/10.1186/1471-2458-13-119

Bunnell, B. E., Beidel, D. C., \& Mesa, F. (2013). A Randomized Trial of Attention Training for Generalized Social Phobia: Does Attention Training Change Social Behavior? Behavior Therapy, 44, 662-673.

Carlbring, P., Apelstrand, M., Sehlin, H., Amir, N., Rousseau, A., Hofmann, S. G., \& Andersson, G. (2012). Internet-Delivered Attention Bias Modification Training in Individuals with Social Anxiety Disorder-A Double Blind Randomized Controlled Trial. BMC Psychiatry, 12, 66.

Compton, R. J. (2000). Ability to Disengage Attention Predicts Negative Affect. Cognition \& Emotion, 1, 401-415. https://doi.org/10.1080/026999300378897

Cristea, I. A., Kok, R. N., \& Cuijpers, P. (2015). Efficacy of Cognitive Bias Modification Interventions in Anxiety and Depression: Meta-Analysis. The British Journal of Psychiatry, 206, 7-16. https://doi.org/10.1192/bjp.bp.114.146761

Dandeneau, S. D., \& Baldwin, M. W. (2004). The Inhibition of Socially Rejecting Information among People with High versus Low Self-Esteem: The Role of Attentional Bias and the Effects of Bias Reduction Training. Journal of Social and Clinical Psychology, 23, 584-603. https://doi.org/10.1521/jscp.23.4.584.40306

Dandeneau, S. D., Baldwin, M. W., Baccus, J. R., Sakellaropoulo, M., \& Pruessner, J. C. (2007). Cutting Stress off at the Pass: Reducing Vigilance and Responsiveness to Social Threat by Manipulating Attention. Journal of Personality and Social Psychology, 93, 651-666. https://doi.org/10.1037/0022-3514.93.4.651

Diener, E., Emmons, R. A., Larsen, R. J., \& Griffin, S. (1985). The Satisfaction with Life Scale. Journal of Personality Assessment, 49, 71-75. https://doi.org/10.1207/s15327752jpa4901_13

Diener, E., Wirtz, D., Tov, W., Kim-Prieto, C., Choi, D., Oishi, S., \& Biswas-Diener, R. (2010). New Well-Being Measures: Short Scales to Assess Flourishing and Positive and Negative Feelings. Social Indicators Research, 97, 143-156. https://doi.org/10.1007/s11205-009-9493-y

Emmelkamp, P. M. (2012). Attention Bias Modification: The Emperor's New Suit? BMC Medicine, 10, 63.

Grafton, B., Ang, C., \& MacLeod, C. (2012). Always Look on the Bright Side of Life: The Attentional Basis of Positive Affectivity. European Journal of Personality, 26, 133-144. https://doi.org/10.1002/per.1842

Hakamata, Y., Lissek, S., Bar-Haim, Y., Britton, J. C., Fox, N. A., Leibenluft, E., Ernst, M., 
\& Pine, D. S. (2010). Attention Bias Modification Treatment: A Meta-Analysis toward the Establishment of Novel Treatment for Anxiety. Biological Psychiatry, 68, 982-990. https://doi.org/10.1016/j.biopsych.2010.07.021

Hallion, L. S., \& Ruscio, A. M. (2011). A Meta-Analysis of the Effect of Cognitive Bias Modification on Anxiety and Depression. Psychological Bulletin, 137, 940-958.

https://doi.org/10.1037/a0024355

Hazen, R. A., Vasey, M. W., \& Schmidt, N. B. (2009). Attentional Retraining: A Randomized Clinical Trial for Pathological Worry. Journal of Psychiatric Research, 43, 627-633. https://doi.org/10.1016/j.jpsychires.2008.07.004

Heeren, A., Mogoașe, C., Philippot, P., \& McNally, R. J. (2015). Attention Bias Modification for Social Anxiety: A Systematic Review and Meta-Analysis. Clinical Psychology Review, 40, 76-90. https://doi.org/10.1016/j.cpr.2015.06.001

Heeren, A., Reese, H. E., McNally, R. J., \& Philippot, P. (2012). Attention Training toward and away from Threat in Social Phobia: Effects on Subjective, Behavioral, and Physiological Measures of Anxiety. Behaviour Research and Therapy, 50, 30-39. https://doi.org/10.1016/j.brat.2011.10.005

Johnson, D. R. (2009). Goal-Directed Attentional Deployment to Emotional FACES and Individual Differences in Emotional Regulation. Journal of Research in Personality, 43, 8-13. https://doi.org/10.1016/j.jrp.2008.09.006

Kruijt, A.-W., Putman, P., \& Van der Does, W. (2013). The Effects of a Visual Search Attentional Bias Modification Paradigm on Attentional Bias in Dysphoric Individuals. Journal of Behavior Therapy and Experimental Psychiatry, 44, 248-254.

https://doi.org/10.1016/j.jbtep.2012.11.003

Li, S., Tan, J., Qian, M., \& Liu, X. (2008). Continual Training of Attentional Bias in Social Anxiety. Behaviour Research and Therapy, 46, 905-912. https://doi.org/10.1016/j.brat.2008.04.005

Lyubomirsky, S., \& Lepper, H. S. (1999). A Measure of Subjective Happiness: Preliminary Reliability and Construct Validation. Social Indicators Research, 46, 137-155. https://doi.org/10.1023/A:1006824100041

MacLeod, C., Mathews, A., \& Tata, P. (1986). Attentional Bias in Emotional Disorders. Journal of Abnormal Psychology, 95, 15-20. https://doi.org/10.1037/0021-843X.95.1.15

MacLeod, C., Rutherford, E., Campbell, L., Ebsworthy, G., \& Holker, L. (2002). Selective Attention and Emotional Vulnerability: Assessing the Causal Basis of Their Association through the Experimental Manipulation of Attentional Bias. Journal of Abnormal Psychology, 111, 107-123. https://doi.org/10.1037/0021-843X.111.1.107

Mathews, A., \& MacLeod, C. (2005). Cognitive Vulnerability to Emotional Disorders. Annual Review of Clinical Psychology, 1, 167-195. https://doi.org/10.1146/annurev.clinpsy.1.102803.143916

Mogg, K., \& Bradley, B. P. (1998). A Cognitive-Motivational Analysis of Anxiety. Behaviour Research and Therapy, 36, 809-848. https://doi.org/10.1016/S0005-7967(98)00063-1

Nelis, D., Kotsou, I., Quoidbach, J., Hansenne, M., Weytens, F., Dupuis, P., \& Mikolajczak, M. (2011). Increasing Emotional Competence Improves Psychological and Physical Well-Being, Social Relationships, and Employability. Emotion, 11, 354-366. https://doi.org/10.1037/a0021554

Neubauer, K., von Auer, M., Murray, E., Petermann, F., Helbig-Lang, S., \& Gerlach, A. L. (2013). Internet-Delivered Attention Modification Training as a Treatment for Social Phobia: A Randomized Controlled Trial. Behaviour Research and Therapy, 51, 87-97. https://doi.org/10.1016/j.brat.2012.10.006 
Pennebaker, J. W. (1982). The Psychology of Physical Symptoms. Berlin: Springer-Verlag. https://doi.org/10.1007/978-1-4613-8196-9

Quoidbach, J., Mikolajczak, M., \& Gross, J. J. (2015). Positive Interventions: An Emotion Regulation Perspective. Psychological Bulletin, 141, 655-693. https://doi.org/10.1037/a0038648

Scheier, M. F., Carver, C. S., \& Bridges, M. W. (1994). Distinguishing Optimism from Neuroticism (and Trait Anxiety, Self-Mastery, and Self-Esteem): A Reevaluation of the Life Orientation Test. Journal of personality and social psychology, 67, 1063-1078. https://doi.org/10.1037/0022-3514.67.6.1063

Schmidt, N. B., Richey, J. A., Buckner, J. D., \& Timpano, K. R. (2009). Attention Training for Generalized Social Anxiety Disorder. Journal of Abnormal Psychology, 118, 5-14. https://doi.org/10.1037/a0013643

Sin, N. L., \& Lyubomirsky, S. (2009). Enhancing Well-Being and Alleviating Depressive Symptoms with Positive Psychology Interventions: A Practice-Friendly Meta-Analysis. Journal of Clinical Psychology, 65, 467-487. https://doi.org/10.1002/jclp.20593

Tamir, M., \& Robinson, M. D. (2007). The Happy Spotlight: Positive Mood and Selective Attention to Rewarding Information. Personality and Social Psychology Bulletin, 33, 1124-1136. https://doi.org/10.1177/0146167207301030

Taylor, C. T., Bomyea, J., \& Amir, N. (2011). Malleability of Attentional Bias for Positive Emotional Information and Anxiety Vulnerability. Emotion, 11, 127-138. https://doi.org/10.1037/a0021301

Wadlinger, H. A., \& Isaacowitz, D. M. (2008). Looking Happy: The Experimental Manipulation of a Positive Visual Attention Bias. Emotion, 8, 121-126.

https://doi.org/10.1037/1528-3542.8.1.121

Wadlinger, H. A., \& Isaacowitz, D. M. (2011). Fixing Our Focus: Training Attention to Regulate Emotion. Personality and Social Psychology Review, 15, 75-102. https://doi.org/10.1177/1088868310365565

Waters, A. M., Pittaway, M., Mogg, K., Bradley, B. P., \& Pine, D. S. (2013). Attention Training towards Positive Stimuli in Clinically Anxious Children. Developmental Cognitive Neuroscience, 4, 77-84. https://doi.org/10.1016/j.dcn.2012.09.004

Watson, D., Clark, L. A., \& Tellegen, A. (1988). Development and Validation of Brief Measures of Positive and Negative Affect: The PANAS Scales. Journal of Personality and Social Psychology, 54, 1063-1070. https://doi.org/10.1037/0022-3514.54.6.1063

Williams, J. M. G., Watts, F. N., MacLeod, C., \& Mathews, A. (1997). Cognitive Psychology and Emotional Disorders (2nd Ed.). Chichester: Wiley. 